\title{
Competição assimétrica e o incremento diamétrico de árvores individuais de Cedrela odorata L. na Amazônia ocidental
}

\author{
Thiago Augusto da CUNHA ${ }^{1}$, César Augusto Guimarães FINGER ${ }^{2}$
}

\section{RESUMO}

Um modelo de crescimento dependente e independente da distância foi ajustado para avaliar como variáveis de competição explicam a variação do crescimento periódico em diâmetro de 132 árvores de cedro (Cedrela odorata). Utilizando a abordagem de crescimento potencial relativo (PRI), estimou-se o incremento relativo em diâmetro (IRd) a partir do produto do crescimento máximo em diâmetro (incremento potencial) e da função modificada (porção do crescimento reduzido). A significância do modelo do crescimento reduzido ajustado demonstrou influência da competiçáo assimétrica sobre o crescimento em diâmetro, explicando $71 \%$ do total da variação e também revelou que a resposta à competição não foi constante, mas variou entre as diferentes classes de tamanho dos indivíduos arbóreos.

PALAVRAS-CHAVE: incremento periódico em diâmetro; índices de morfometria; índices de competiçấo.

\section{Asymmetric competition and the diameter increment of Cedrela odorata $\mathrm{L}$. trees in western Amazonia.}

\section{ABSTRACT}

A dependent and independent growth distance model was fitted to evaluate how competition variables may account for the variation of periodic diameter increment of 132 Cedrela odorata trees. Using a Potential Relative Increment (PRI), the relative diameter increment (IRd) was estimated from the multiplication of maximum diameter growth (potential increment) by modified function (portion of the reduced growth due to competition by light and growth space). The significance of the reduced fitted model demonstrated the influence of asymmetric competition variables on diameter growth, explaining $71 \%$ of the total variation as well as indicated that competition responses were not constant, but it varied among the different size classes of trees.

KEYWORDS: diameter periodic increment; morphometric indices; competition indices.

\footnotetext{
1 Programa de Pós-graduação em Engenharia Florestal, Universidade Federal de Santa Maria, CEP 97105-900, Santa Maria (RS), etsfor@yahoo.com.

2 Programa de Pós-graduação em Engenharia Florestal, Universidade Federal de Santa Maria, CEP 97105-900, Santa Maria (RS), caesar.finger@gmail.com, cesar.finger@ufsm,br.
} 


\section{INTRODUÇÃO}

A floresta Amazônica apresenta uma grande diversidade de espécies arbóreas, potenciais para a produção de madeira. Logo, há um considerável interesse em promover a sustentabilidade da produçáo de madeira como uma estratégia para a conservação e a manutenção da produção de madeira serrada. Certamente, aplicações de práticas de manejo florestal, tais como a seleção de espécies para produção de madeira; estimativas de ciclos de corte orientados (cf. Schöngart 2008) e a prescrição de tratamentos silviculturais específicos, constituem ferramentas que podem contribuir com uma floresta sustentável, do ponto de vista da produçấo de madeira. Contudo, para colocar isto em prática assume-se como informação mínima conhecer o processo de regeneração, mortalidade, recrutamento e, o mais importante, descrever o padrão de crescimento das espécies de interesse (Vanclay 1994).

O estudo envolvendo o crescimento de árvores individuais na Amazônia se encontra, ainda, em seus primórdios, com um número limitado de modelos de crescimento em publicaçóes esparsas (p.e., da Cunha 2009). Contribuir com o estabelecimento do manejo racional da florestal exige dados confiáveis de crescimento. Embora seja antiga a controvérsia sobre a formação de anéis anuais em madeiras tropicais, devido a ausência de estaçôes climáticas marcadas (Lieberman e Lieberman 1985), as análises de anéis de crescimento em florestas tropicais vêm ganhando credibilidade com estudos que comprovam a existência para algumas espécies de interesse comercial, como Cedrela odorata e Swietenia macrophylla, ambas as espécies da família Meliaceae (Dünisch et al. 2003; Worbes 1999). Portanto, isso viabiliza a aplicação de métodos dendrocronológicos para obter dados de crescimento para modelagem.

Uma das possibilidades de modelar o incremento em diâmetro de uma árvore é utilizando um modelo composto ou a abordagem de modelagem potencial/modificado (Hasenauer 2006). O modelo composto descreve o incremento em funçấo de variáveis de atributo da árvore como a morfometria da copa, espaço de crescimento e disponibilidade de luz solar. Essas variáveis são consideradas ponto de partida para a descrição matemática do crescimento de árvores (Wykoff 1990; Choi et al. 2001). Por outro lado, o modelo potencial/ modificado considera que o crescimento de uma árvore é o resultado do produto de seu crescimento potencial com uma porção reduzida do crescimento denominada de função modificada (Holdaway 1984). A funçáo modificada representa o crescimento das árvores sob o efeito da competição que reduz o crescimento potencial (máximo) para o crescimento esperado (Leary e Holdaway 1979; Quicke et al. 1994). A interação entre árvores (aqui referida como competição ou concorrência) é um fator importante no campo florestal; entretanto, definir qual método de análise e interpretação a utilizar é uma questão em aberto, principalmente em área de florestas da amazônia.

Nesse contexto, por meio da análise de anéis de crescimento, reconstruiu-se o diâmetro de árvores individuais de cedro (Cedrela odorata) e utilizando a modelagem potencial/modificada ajustou-se uma equaçáo com o objetivo de responder como o crescimento em diâmetro é influenciado por árvores vizinhas competidoras. A equação ajustada poderá ser empregada como ferramenta de planejamento em floresta tropical.

\section{MATERIAL E MÉTODOS}

\section{Coleta dos dados}

Um total de 132 árvores de cedro (denominadas de árvores-objetivo) foi amostrado em Unidades de Produçáo Anual (UPA) de três Planos de Manejo Florestal Sustentável no município de Porto Acre, estado do Acre. A área total das UPAs é de 973 ha. A floresta caracteriza-se com floresta de terra-firme, com árvores formando três ou mais estratos, dossel médio entre 25 a 35 metros e indivíduos emergentes alcançando 45 metros de altura. Em média ocorrem 111 espécies por hectare para árvores com DAP $\geq 10$ (diâmetro medido a $1,30 \mathrm{~m}$ do solo) e a área basal média, considerando árvores com DAP $\geq 20 \mathrm{~cm}$, é de $15 \mathrm{~m}^{2} /$ ha (Acre 2000).

Em cada uma das árvores-objetivo dois rolos de incremento (baguetas) foram extraídos radialmente à altura de $1,30 \mathrm{~m}$ (d) com trado Pressler (Häglof Mora 5,15 mm de diâmetro). Em árvores com raízes tabulares (sapopemas) as baguetas foram extraídas a uma altura de $30 \mathrm{~cm}$ acima para prevenir super/ subestimação na taxa de crescimento (p.e. Metcalf et al. 2009). Os anéis de crescimento foram visualmente cruzados (Stokes e Smiley 1996) e o crescimento anual foi obtido pela soma do incremento radial medido nas duas baguetas com precisão de $0,01 \mathrm{~mm}$ utilizando mesa micrométrica Lintab II (Frank Rinn S.A. Heidelberg, Germany) e software TSAP-Win (Rinn 2003). Portanto, o diâmetro foi reconstruído para cada i-ésima árvore-objetivo.

Todas as árvores foram estratificadas em classes de diâmetro com intervalo de $10 \mathrm{~cm}$ com aproximadamente o mesmo número de árvores dentro de cada classe (distribuição uniforme). Desta forma, foi possível verificar a influência de diferentes índices de competição por classe de tamanho das árvores. O índice de iluminação de copa também foi avaliado para cada árvore-objetivo (modificado de Dawkins 1963 e adaptado por Silva et al. 1995; Figura 1): PS1 indicando copas que recebem luz plena de cima e dos lados (dominante); PS2 copas com luz plena de cima e lateralmente sombreadas (intermediária) e PS3 para copas sem iluminação direta (dominada). 


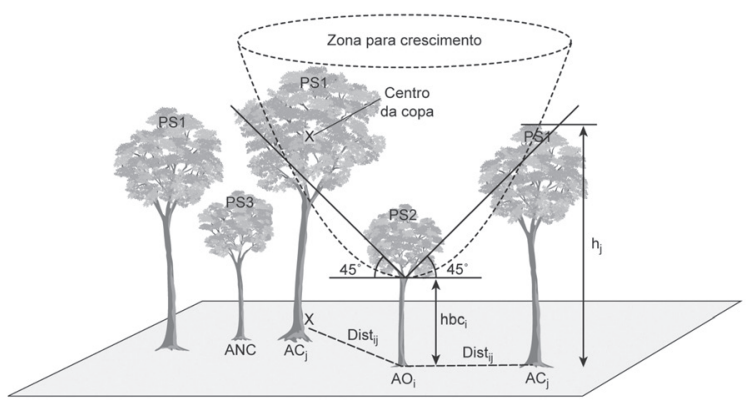

Figura 1 - Critério para a seleção de árvores competidoras (ACi). A altura hj foi corrigida em situações de inclinação do terreno (eixo Z).

Variáveis discretas tipo Dummy foram utilizadas para incluir o efeito de variáveis qualitativas (classe de diâmetro e posição social da copa) na equação de regressão.

\section{Estrutura do modelo de crescimento}

Neste estudo utilizou-se a aproximação da modelagem potencial/modificada (Equação 1) com três componentes. Esse modelo possibilitou descrever o crescimento periódico de forma independente da idade utilizando dados empíricos. Zhang et al. (1997) considera que esse tipo de modelagem explica melhor o conceito biológico do crescimento.

$$
\left.\left[\begin{array}{l}
\text { Incermento } \\
\text { diamétrico }
\end{array}\right]=[\text { Incremento potencial }] \times \text { [Função modificada }\right]
$$

Justifica-se este cenário preditor como consistente já que o crescimento potencial é reduzido pelo efeito da competição.

\section{Incremento diamétrico}

Os dados de incremento diamétrico da Equação 1 foram obtidos a partir da reconstruçáo do diâmetro inicial $\left(d_{t-4}\right)$. Assim utilizando o diâmetro atual $(d)$ obteve-se a taxa periódica de mudança do diâmetro (Equação 2) (Bragg 2001) denominada de Incremento Relativo em diâmetro:

$$
I R d=\frac{d_{t}-d_{t-4}}{d_{t-4}}
$$

O IRd expressa a eficiência do crescimento para um mesmo diâmetro sendo uma medida sensível de resposta da árvore frente aos efeitos da competição.

Desde que o incremento em diâmetro e o incremento em área basal são matematicamente relacionados, a decisão em modelar qualquer um dos incrementos é baseado em conveniência, já que nenhum deles causa diferenças na acurácia em ajuste (West 1995) e qualquer diferença observada está atribuída à estrutura do erro (Vanclay 1994).

\section{Incremento potencial}

Os valores de $I R d$ foram agrupados em classes de diâmetro com intervalo de $5 \mathrm{~cm}$ e os maiores valores de incremento, para cada classe, foram separados gerando um subconjunto de dados que representou o crescimento de árvores sem concorrência (MAXIRd). Para descrever MAXIRd utilizouse a função potencial sugerida por Bragg (2001) (Equação 3). Com esta metodologia foi possível estimar o máximo incremento em diâmetro $\left(I P d_{\max }\right)$ numa previsão de quatro anos (Equação 4).

$$
\begin{gathered}
M A X I R d=\beta_{0} \times d_{\max }^{\beta_{1}} \times \beta_{2}^{d_{\max }} \\
I \hat{P}_{\max }=d \times M A \hat{X} I R d
\end{gathered}
$$

Onde: $M A X I R d=$ incremento relativo potencial em diâmetro; $d_{\max }=$ DAP das árvores com máximo IRd dentro de cada classe de diâmetro; $b_{0}, b_{1}, b_{2}=$ coeficientes de regressão.

\section{Função modificada}

A função modificada foi utilizada para representar a porção reduzida do crescimento em diâmetro (PRCd) relacionado a fatores de competição, definido pelos índices de competição e dimensão da copa. Este componente do modelo foi obtido pela Equação 5:

$$
[\text { PRCd }]=\left[\begin{array}{l}
\text { Incrementio } \\
\text { diamétrico }
\end{array}\right] /[\text { Incremento potencial estimado }]
$$

Esta função tem escala variando de zero a 1 em que valores igual a 1 indicam nenhuma redução no crescimento das árvores e valores abaixo de 1 e próximos de zero indicam a porção reduzida do crescimento que está relacionado a resposta da árvore-objetivo à disposição de recursos no meio modificado por fatores de competição.

\section{Efeito da competição}

Para determinar a relação entre variáveis de competição sobre o crescimento, a variável PRCd foi modelada em função de variáveis que medem a situação atual da competição representado pelos índices de competição e índices morfométricos que expressam o efeito da competiçáo no passado (Equação 6).

$$
\text { PRCd = (índices de competição)X( índices Morfétricos) }
$$

Neste cenário de predição, o crescimento em diâmetro exibe um decréscimo com o aumento da competição atual exercido pelas árvores vizinhas e com a competição sofrida no passado representado pela morfometria da copa.

O status competitivo e atributos de vigor de cada árvore (p.e. diâmetro e comprimento da copa) não foram reconstruídos já que se assumiu, na maioria dos casos, que estas variáveis mudam em pequenas quantidades ao longo do tempo e que o erro causado por esta consideração é insignificante para curtos períodos de tempo. Entretanto, pode haver discordância no grau de plasticidade da copa em que 
mudanças consideráveis podem ocorrer no estado inicial de sucessão (pequenos diâmetros; Muth e Bazzaz 2002).

\section{Seleção de árvores competidoras}

Para avaliar a hipótese de competição por luz (competição vertical) empregou-se o conceito de competição assimétrica em nível individual (Schwinning e Weiner 1998; Freckleton e Watkinson 2001), em que as árvores de cedro estavam (one-sided) ou não submetidas ao efeito da competição por árvores vizinhas competidoras. Assim, a interação foi calculada considerando um processo espacial considerando a alturas das árvores. Desta forma, considerou-se um competidor potencial qualquer árvore vizinha com sua copa dentro da zona de crescimento virtual formada por um ângulo vertical de $45^{\circ}$ (veja Figura 1). Portanto, a probabilidade de uma árvore vizinha ser uma competidora aumenta com o aumento de seu tamanho, representado pela altura total $\left(b_{j}\right)$, e a proximidade da árvore-objetivo $\left(\right.$ Dist $\left._{i j}\right)$. Na prática o uso desta metodologia em campo torna-se fácil aplicando a Equação 7 :

$$
\operatorname{tg} 45^{\circ}=h_{j}-h b c_{i} / \text { Dist }_{i j}=1 \Rightarrow\left\langle\operatorname{Dis}_{i j} \leq h_{j}-h b c_{i}\right\rangle
$$

Onde: $h b c_{i}=$ altura na base da copa; $i$ e $j=i$-ésima árvoreobjetivo e a j-ésima árvore competidora, respectivamente.

Esta metodologia de seleçáo de competidores incluiu a suposição de que o stress competitivo experimentado pela árvore-objetivo é uma funçâo do grau com o que a "zona de crescimento" é sobreposta pelas árvores vizinhas (Daniels 1976). Vale notar que, para árvores competidoras inclinadas, a distância Dist tij foi considerada a partir do centro da copa da mesma (ponto x da Figura 1) e não da base do tronco.

\section{Índices de competição avaliados}

Em cada árvore-objetivo e em seus respectivos competidores foram medidos variáveis dendrométricas de tamanho (d e h) e a distância entre a árvore-objetivo e a cada competidora (Dist $\mathrm{tij}_{\mathrm{ij}}$ ) dispondo de informação sobre a influencia da distribuiçẫo espacial. Desta forma, quantificou-se o status competitivo de cada árvore-objetivo utilizando os índices de competição da Tabela 1.

A relaçẫo Comp1 calcula a competição que uma ou mais árvores competidoras exercem sobre a árvore-objetivo em base a um ângulo vertical implicando maior concorrência quanto maior seu valor. Outro índice utilizado amplamente é o Comp3 que pondera a relação de diâmetros com a distância implicando numa maior concorrência quanto mais próximos e grandes sejam os competidores. $\mathrm{O}$ índice 2 é semelhante ao índice 3 mas utiliza a altura total das árvores. $\mathrm{O}$ índice Comp4 calcula a concorrência relacionando o diâmetro da árvore-objetivo $\left(\mathrm{d}_{\mathrm{i}}\right)$ com o diâmetro das competidoras desconsiderando a distância entre ambas. Assim, quanto maior
Tabela 1- Índices de competição (Comp) utilizados.

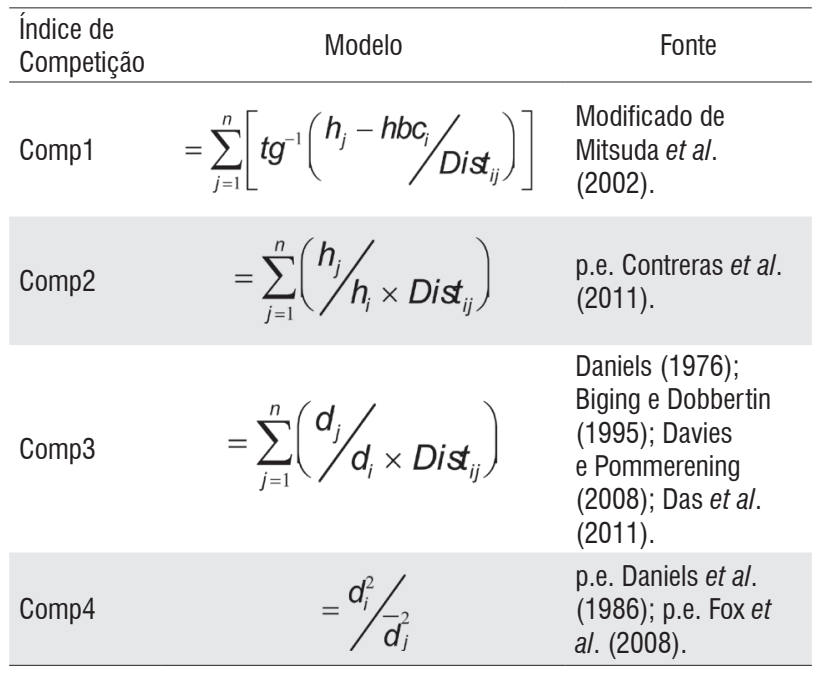

Em que: d, h, hbc, e Dist é o diâmetro a altura do peito, altura total, altura do tronco até a base da copa e distância horizontal; $n=$ todas as árvores interceptadas pela linha de $45^{\circ}$; i e j=i-ésima árvore-objetivo e a j-ésima árvore competidora.

o aumento do valor do índice indica uma menor concorrência. De acordo ao método de seleçáo de competidores, árvores sem efeito da concorrência apresentaram valores zero e não negativos.

\section{Competição no passado}

O termo competiçáo passada considerou o efeito da competição na qual a árvore foi submetida no passado e que interferiu nas dimensóes das copas apresentadas. Para tal, assumiu-se a hipótese de que os índices morfométricos refletem o efeito da competição no passado ocasionando a mudanças interdimensionais da árvore e, portanto, variaçóes na captaçáo de recursos para o crescimento (Clark e Clark 2001). Desta forma, para duas árvores de mesmo diâmetro assumiu-se que apresentou considerável grau de competição a árvore com menor diâmetro de copa. Logo, a redução na taxa de crescimento pode proceder da redução no tamanho da copa como consequência do efeito da competiçáo em anos anteriores.

Índices de morfometria foram calculados utilizando as expressóes da Tabela 2. O índice morfométrico 1 (Morf1, denominado de saliência da árvore) descreveu o vigor entre árvores com o mesmo diâmetro e indica o espaço necessário para o crescimento da árvore à medida que cresce em diâmetro. Assim, a diminuição do seu valor pode indicar forte competiçáo experimentada pela árvore.

O índice de abrangência (Morf2), calculado pela razão $\mathrm{dc} / \mathrm{h}$ também indica a necessidade de espaço da árvore com o aumento da altura. Desta forma, para uma mesma altura total, atingirá maior espaço o indivíduo que tiver maior diâmetro de copa. 
Tabela 2 - Índices morfométricos (Morf) que descrevem o efeito da competição passada

\begin{tabular}{|c|c|c|}
\hline Índice morfométrico & Modelo & Fonte \\
\hline Morf1 & $=d c / d$ & Assmann (1970) \\
\hline Morf2 & $=d c / h$ & Assmann (1970) \\
\hline Morf3 & $=d c / c c$ & $\begin{array}{l}\text { Durlo e Denardi (1998); } \\
\text { Sterba et al. (2002) }\end{array}$ \\
\hline Morf4 & $=c c / h$ & p.e. Vettenranta (1999) \\
\hline Morf5 & $=d c^{2} / d^{2}$ & Assmann (1970) \\
\hline Morf6 & $=h / d$ & Assmann (1970) \\
\hline Morf7 & $=\sqrt{d c \times h^{-0,5}}$ & $\begin{array}{l}\text { p.e. Monserud e Sterba } \\
\text { (1996); Schröder et al. } \\
\text { (2002). }\end{array}$ \\
\hline
\end{tabular}

Em que: $\mathrm{dc}=$ diâmetro de copa derivado de oito raios realizada em direções cardiais e intercardiais (Alder 1980); $\mathrm{h}=$ altura total; $\mathrm{cc}=$ comprimento de copa.

O índice Morf3 (Formal de copa) calculado pela razão $\mathrm{dc} / \mathrm{cc}$ expressa a capacidade fotossintética (produtividade) da árvore. Em geral, quanto menor seu valor, maior é a influência das árvores vizinhas na expansão lateral da copa da árvore considerada.

A proporção de copa (Morf4) mede o potencial fotossintético da árvore e é sensível aos efeitos da competiçấo diminuindo o comprimento da copa sendo, por esta razão, altamente correlacionado com o crescimento (Daniels et al. 1986).

\section{Adequação do modelo ajustado}

Utilizou-se análise de regressão utilizando o método Stepwise para examinar relaçôes entre variáveis de competição e incremento em diâmetro das árvores. Critérios de diagnóstico baseados nos dados observados e estimados foram calculados para determinar o nível de ajuste do modelo (Tabela 3). Incluiu-se o critério de informaçáo de Akaike para medir a falta de ajuste do modelo penalizada pelo número de parâmetros (Porçáo 2xp). Para o controle da multicolinearidade eliminouse variáveis, previamente selecionadas, que apresentaram fator de inflação da variância (VIF) maiores do que 10.

Os pressupostos de constância da variância e normalidade dos resíduos foram avaliados utilizando os testes de White e Kolmogorov-Smirnov, respectivamente. Todas as análises estatísticas foram realizadas no sistema estatístico SAS V9.2 (SAS/Stat, 2004) utilizando o procedimento REG e GLM, considerando nível de significância $\mathrm{p}<0,05$.
Tabela 3 - Critérios para diagnóstico do modelo de regressão.

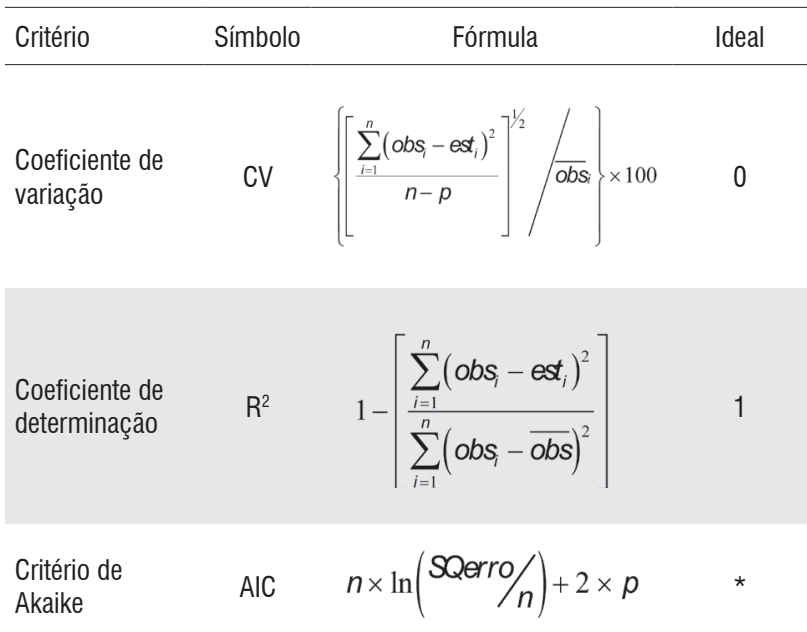

Em que: $n=$ número de observacões; $p=$ número de parâmetros; SQerro=soma de quadrados do erro; In=logaritmo natural; * = quanto menor o valor melhor será 0 modelo ajustado.

\section{RESULTADOS E DISCUSSÃO}

\section{Crescimento potencial}

Vários indivíduos apresentaram crescimento potencial ao longo de toda a amplitude de diâmetro (25 indivíduos com máximo IRd do total de 132 árvores avaliadas) com variação considerável dentro da mesma classe (Figura 2a). Esse efeito reflete, possivelmente, a dinâmica do dossel atribuído a fatores de redução do crescimento (p.e. competiçáo) e comumente observado em florestas nativas.

A condição de crescimento potencial foi descrita pela curva média ajustada pela Equação 3 (Figura 2b). A aplicação desse modelo pode apresentar algumas dificuldades na determinação do crescimento potencial (MAXIRd). Bragg (2001) sugeriu que existem duas fontes de erro: (1) erro nos dados que se refere aos outliers e (2) representação das classes de diâmetro (inadequância da amostragem) o que poderia resultar na subestimação das condiçôes ótimas de crescimento determinadas pela curva ajustada (Figura $2 \mathrm{~b}$ ) principalmente em árvores de grande porte que, geralmente, apresentam poucos indivíduos amostrados.

Multiplicando os resultados da Equação 3 pelo diâmetro atual obteve-se a curva enviesada (Figura 2c) que representou a máxima taxa de incremento periódico em diâmetro sob condiçôes ótimas de crescimento. Esta curva revelou que mesmo indivíduos de grande porte (p.e. DAP de $90 \mathrm{~cm}$ ) agregaram, em média, $1 \mathrm{~cm}$ em diâmetro com o máximo de incremento de $6,4 \mathrm{~cm}$ ocorrendo em árvores de $50 \mathrm{~cm}$ de diâmetro no período de quatro anos (cf. Figura 2c). 

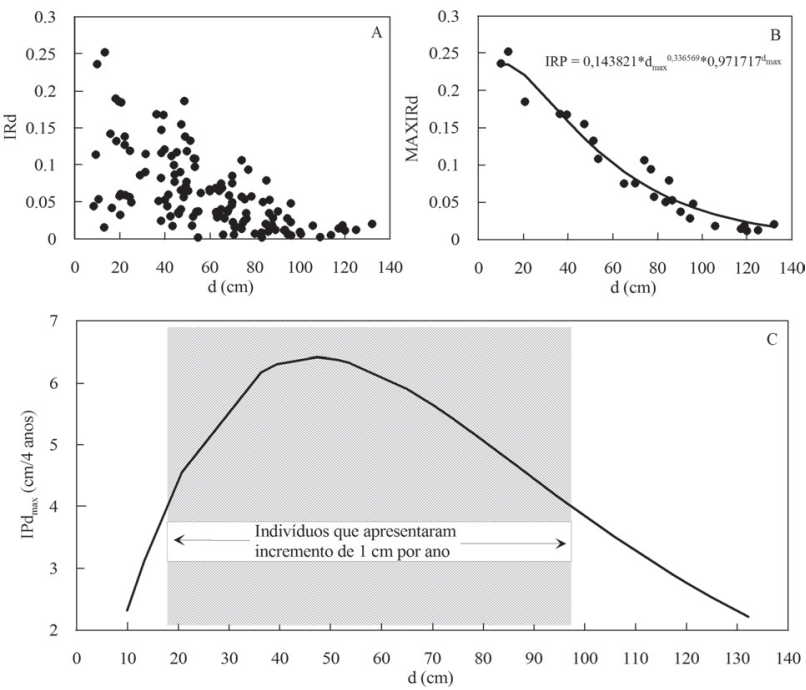

Figura 2 - Incremento relativo em diâmetro (a), taxa máxima de crescimento observada em 25 indivíduos e a curva ajustada (b) utilizando a Equação 3, incremento máximo em diâmetro (c) todos em função do diâmetro atual obtido pela Equação 4.

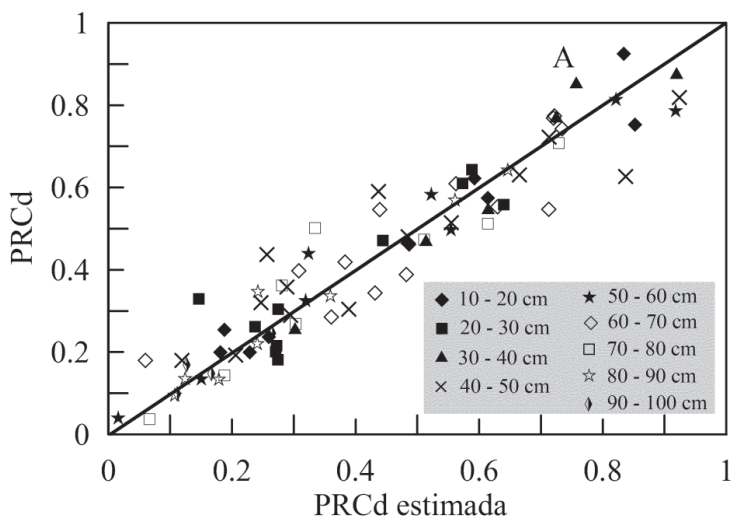

que o ângulo para os valores estimados versus observados foi igual a 1. Este efeito refletiu a boa distribuição dos dados de incremento diamétrico estimados sobre os observados (Figura 3b).

A maioria das variáveis independentes incluídas na equação teve alto grau de significância que explicaram 71\% (coeficiente de determinação ajustado) da variação total da porção reduzida do crescimento (PRCd). A maior parte da variação explicada pelo modelo decorre de variáveis que refletem a competiçáo passada (índices morfométricos incluindo variáveis de altura total e a área de projeção da copa). O status competitivo, representado pelos índices de competição, também explicou a variação na PRCd.

A equação também revelou que a resposta à competição não foi constante, mas variou entre os diferentes portes das árvores (revelado pela significância das variáveis Dummy para classe de diâmetro). O índice de competição 4 atuou em árvores consideradas pequenas quanto em médias (cf. Tabela 4).

Figura 3 - Porção reduzida do crescimento observado e estimado por classe de diâmetro (a) e crescimento atual relativo em função do diâmetro (b) obtido pela Equação 1.

\section{Capacidade de estimativa do modelo}

Sob as condições utilizadas para a seleção do modelo, a modelagem da PRCd indicou relação significativa tanto para variáveis que expressam a competição atual e passada. A estratificação dos dados em diferentes classes de diâmetro e posição social levou a uma atuação especifica das variáveis de competiçáo sobre o crescimento das árvores (Tabela 4). Este procedimento produziu maior eficiência em descrever a grande variação do crescimento que foi observada principalmente nas menores classes de DAP (Figura 3a classes menores a 40-50 cm) que apresentaram leve desvio referente à linha de referência. Para classes de tamanho maiores (Figura 3a classes a partir de $50-60 \mathrm{~cm}$ ) o modelo foi menos tendencioso. Em geral, o modelo produziu estimaçôes não tendenciosas já

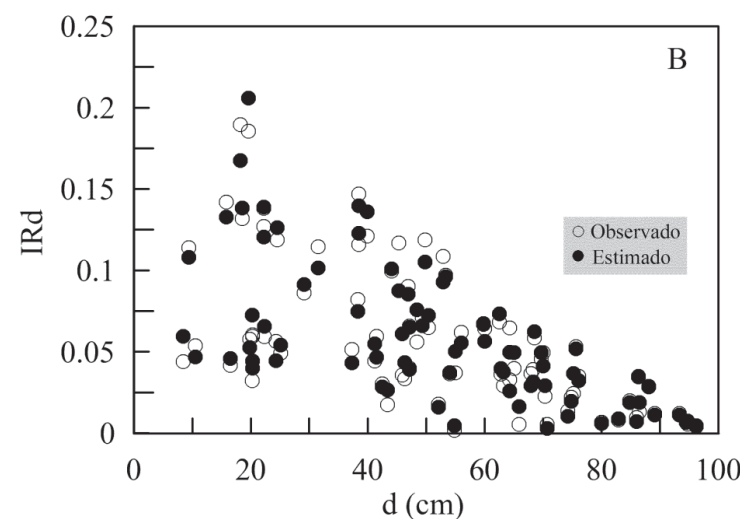
Diagnóstico do modelo ajustado

Os gráficos de resíduos absoluto (abs) e padronizado (pad) da Figura 4 revelaram padrão aleatório de distribuição. A heterocedasticidade foi descartada pelo teste de White (P $=0,6813)$. Este resultado reflete a causa-efeito do número de variáveis preditoras (transformaçóes, interaçóes e combinaçóes com variáveis Dummy) incluídas no modelo de regressão, capaz de abranger toda a variação do crescimento principalmente para indivíduos de pequeno porte.

Os efeitos das distintas variáveis de competição na equação foram aparentemente aleatórios conforme demonstrado pelo gráfico de probabilidade normal (Figura 4). Apenas duas estimaçóes revelaram ser altamente influentes, detectadas pela distância de Cook (Figura 4). 
Tabela 4 - Modelo de regressão para estimar a porção reduzida do crescimento em diâmetro PRCd para árvores de cedro.

\begin{tabular}{|c|c|c|}
\hline Variável independente & Coeficiente de regressão & Descrição \\
\hline Intercept & $-0,2885^{*}$ & \\
\hline D2Morf5 & $-1,9444^{* *}$ & Índice morfométrico 5 significativo para árvores entre $20-30$ cm de diâmetro (d). \\
\hline D4Projcopa & $-0,0015^{* * *}$ & Projeção da copa significativo para árvores entre $40-50$ cm de diâmetro (d). \\
\hline D5Morf3 & $1,2290^{* * *}$ & Índice morfométrico 3 significativo para árvores entre 50-60 cm de diâmetro (d). \\
\hline D5Morf5 & $-9,8618^{*}$ & Índice morfométrico 5 significativo para árvores entre 50-60 cm de diâmetro (d). \\
\hline D5Morf7 & $-2,8076^{* * *}$ & Índice morfométrico 7 significativo para árvores entre 50-60cm de diâmetro (d). \\
\hline D6Projcopa & $-0,0018^{* * *}$ & Projeção da copa significativo para árvores entre 60-70 cm de diâmetro (d). \\
\hline D7Morf3 & $-0,1867^{* * *}$ & Índice morfométrico 3 significativo para árvores entre $70-80$ cm de diâmetro (d). \\
\hline D8Morf7 & $-4,5117^{\star * *}$ & Índice morfométrico 7 significativo para árvores entre 80-90cm de diâmetro (d). \\
\hline D12Morf6 & $1,6083^{\star \star \star}$ & Índice morfométrico 6 significativo para árvores em posição social dominante. \\
\hline $\mathrm{D} 13 \mathrm{H}$ & $0,0139^{*}$ & Altura total significativa para árvores em posição social intermediária. \\
\hline D13Morf4 & $0,9521^{\star \star}$ & Índice morfométrico 4 significativo para árvores em posição social intermediária. \\
\hline D14Morf2 & $1,7527^{\star \star *}$ & Índice morfométrico 2 significativo para árvores em posição social dominada. \\
\hline D14Morf5 & $-4,6316^{*}$ & Índice morfométrico 5 significativo para árvores em posição social dominada. \\
\hline D14Morf6 & $0,2985^{*}$ & Índice morfométrico 6 significativo para árvores em posição social dominada. \\
\hline D5Comp1 & $-0,0013^{*}$ & Índice de competição 1 significativo para árvores entre 50-60 cm de diâmetro (d) \\
\hline D8Comp3 & $-0,3555^{*}$ & Índice de competição 3 significativo para árvores entre $80-90 \mathrm{~cm}$ de diâmetro (d) \\
\hline D2Comp4 & $-0,1440^{*}$ & Índice de competição 4 significativo para árvores entre $20-30 \mathrm{~cm}$ de diâmetro (d) \\
\hline D6Comp4 & $-0,1106^{*}$ & Índice de competição 4 significativo para árvores entre $60-70 \mathrm{~cm}$ de diâmetro (d) \\
\hline gl & 112 & 71,0 \\
\hline CV & 30,1 & $-303,2$ \\
\hline
\end{tabular}

Valores de significância são mostrados para cada variável preditora ${ }^{*} \mathrm{P}=0,05-0,01$; $* * \mathrm{P}=0,01-0,001 ;{ }^{*} * \mathrm{P}<=0,001$. $\mathrm{g} \mid=$ graus de liberdade do erro.

\section{Análise dos índices de competição}

Os índices de competição utilizados foram desenvolvidos e testados em várias pesquisas anteriores (veja fontes na Tabela 1). Entretanto, por serem simples expressóes matemáticas não necessariamente representam todo o processo ontogênico da competiçáo. Logo, quando uma árvore apresenta pequeno porte, seja em diâmetro, altura total e diâmetro da copa, em relação às árvores vizinhas assumiu-se que apresentou, possivelmente, falta de vigor competitivo (Davis e Johnson 1987). Em floresta tropical, entretanto, estas características são comumente observadas em indivíduos em boas condições de crescimento (posição social dominante ou intermediária) causando baixo poder preditor para os índices.

Vários aspectos devem ser considerados antes de interpretar a significância dos índices de competição comprovada pela equação ajustada. Primeiro, os índices variam de acordo ao 

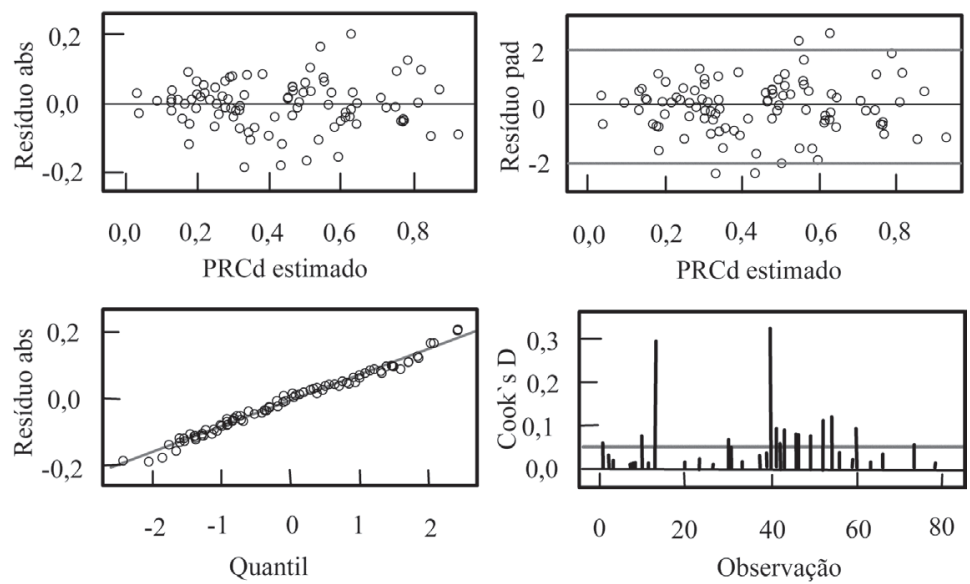

Figura 4- Distribuição residual não heterocedástica obtidos da Equação do crescimento reduzido (Modelo 5).

método de cálculo da competição. Devido serem simples expressões matemáticas e, não necessariamente, representarem todo o complexo processo ontogênico das condiçôes de competição, que facilmente são alteradas, o que pode resultar numa relação matemática inteiramente marginal (Larocque 2002). A maioria dos estudos de competição entre árvores tem focado no desempenho médio (p.e. crescimento diamétrico) em relação com a densidade do povoamento (p.e., uso do índice de BAL). Entretanto, utilizar somente a densidade como uma medida da competição para avaliar o desempenho das árvores em floresta mista, como na Amazônia, pode resultar em modelos seriamente tendenciosos, já que os efeitos da variação, que pode ser grande, são mascarados. Isso reforça a cautela em considerar o uso de modelos não espaciais (p.e. Índice de Glover e Holl) na modelagem do crescimento de florestas mistas. Ademais, a concorrência espacial calculada para povoamentos equiâneo, puro e manejado, depende necessariamente da idade do povoamento, já que com o desbaste a distância absoluta entre as árvores aumenta. Para o cenário Amazônico, onde não ocorrem desbastes, os índices espaciais podem apresentar outro comportamento tornando a habilidade estimativa dependente destas variaçóes, causando, assim, baixo poder explicativo. Esta razão pode explicar porque modelos de crescimento não têm sido extensamente utilizados durante a planificação do manejo florestal.

Segundo, os índices variam de acordo ao método de seleção de indivíduos competidores. Uma das razôes de que os índices de competição podem falhar em representar a concorrência pode estar atribuída à área ao redor da árvore-objetivo utilizada para a amostragem dos competidores. Definir uma zona exata em que ocorre a influência de árvores vizinhas competidoras é bastante difícil (Tonini 2007). Entretanto, Vettenranta (1999) sugere que realmente não há uma razão natural, pois a competição cessaria repentinamente a uma determinada distância, sendo mais realista assumir que a competição decresce com o aumento da distância à árvore-objetivo. Para isto, Daniels (1976) relatou a vantagem de utilizar o método de área variável (Goniométrico) para seleção de indivíduos competidores o que produziu maiores valores de correlação com o crescimento periódico em diâmetro do que quando se utilizou raios fixos. Devido a isto, métodos efetivos para medir a competição foram criados, entretanto, em nenhum dos índices submetidos a essa análise, a competição foi expressa em termos de taxa de crescimento efetivo, mas utilizou o efeito na dimensão da árvore. Boivin et al. (2010) relataram aumento do poder preditivo do modelo de crescimento quando incluído no cálculo da competição o sombreamento da árvore-objetivo causado pela árvore vizinha competidora. Esta melhora pode estar associada ao fato que o resultado da interação (competição) determina o tamanho da copa de cada árvore individual, o que reflete na interceptação de luz, capacidade fotossintética, crescimento e até mesmo sobrevivência (Thorpe et al. 2010).

Os índices de competição espaciais quando utilizados em povoamentos equiâneos são considerados dependentes da idade, já que com o aumento da idade a distância absoluta entre as árvores também aumenta (p.e. desbaste). Desta forma, em floresta tropical o índice pode apresentar outro comportamento tornando a sua habilidade explicativa dependente destas variaçóes.

Por esta razão, para descrever a competição, Zhao et al. (2004) relatam que, para florestas mistas, é preferível utilizar modelos de competição dependentes da distância, já que o efeito de árvores vizinhas sob o crescimento da árvoreobjetivo decresce com a distância. Entretanto, alguns autores recomendam incluir na modelagem, índices de competição que desconsideram o espaço (p.e. Fox et al. 2008).

O índice de competição Glover e Holl (Comp4) foi amplamente recomendando para determinar a competição de povoamentos homogêneos de mesma idade, tamanho e crescimento potencial (Dimov et al. 2008), devido à vantagem 
de ser independente do espaço e explicar boa parte da variação do crescimento. Entretanto, o índice pode perder sua funcionalidade em povoamentos mistos de diferentes idades onde comumente encontram-se indivíduos competidores de menor diâmetro (elevada relação h/d).

No contexto competitivo, o modelo demonstrou efeito significativo da concorrência que levou à redução do crescimento em diâmetro. Este efeito está em consonância com a literatura que demonstrou tais relaçóes em florestas tropicais (Foli, 1993) e em florestas temperadas (Biging e Dobbertin 1995; Monserud e Sterba 1996; Sterba et al. 2002).

Contrário à expectativa, a análise não demonstrou evidências de aumento do status competitivo com a diminuição do porte das árvores. Para alguns indivíduos pequenos $(10<\mathrm{d}$ $<20)$ os índices de competição estimaram fraca ou nenhuma competição (valores próximo de zero para Comp1, Comp3 e altos valores para Comp4). Portanto, considerando que a luz é considerada o fator de maior importância para o crescimento, podemos inferir que árvores pequenas com o mesmo diâmetro receberam este recurso em diferentes níveis que provavelmente resultou em diferentes níveis de redução do crescimento (variação para os valores de PRCd). Este fato pode esclarecer a significância de variáveis de competição combinadas com variáveis Dummy para diferentes classes de diâmetro. Do contrário, para árvores grandes $(\mathrm{d}>70 \mathrm{~cm}) \mathrm{o}$ resultado seguiu a expectativa.

\section{CONCLUSÕES}

Este estudo apresentou uma abordagem de modelagem que pode ser utilizada para estimar o crescimento periódico em diâmetro (IRd) de árvores individuais de Cedrela odorata baseado em variáveis que expressam a concorrência. Em termos de eficiência e consistência, o modelo apresentou boas estatísticas de avaliação revelando que variáveis que expressam a competição passada e o status competitivo englobam grande parte dos efeitos que moldaram a variação observada do crescimento em diâmetro.

Embora os modelos de crescimento em diâmetro seja um componente fundamental para planejar o crescimento das florestas em muitos sistemas de predição da produção, sua aplicabilidade pode ser restrita sem informação sobre a mortalidade e regeneração da floresta. Finalmente, pela análise de anéis de crescimento é possível quantificar o crescimento periódico em diâmetro para árvores de cedro, tornando uma ferramenta útil para a modelagem do crescimento florestal na Amazônia.

\section{AGRADECIMENTOS}

Agradecemos à CAPES, órgão federal de relevante importância, pela concessão de bolsa de estudo sanduiche a qual apoiou a concretização deste artigo.

\section{REFERÊNCIAS BIBLIOGRÁFICAS}

Acre. 2000. Natural-Economic Zoning: resources and environment, Governo do Estado do Acre. Rio Branco, BRA. 178 pp (in Portuguese)

Alder, D. 1980. Estimación del volume forestal y predicción del redimiento: con referencia especial a los trópicos. FAO: MONTES, Roma. 80 pp.

Assmann, E. 1970. The principles of forest yield study. Perfamon Press, Oxford, USA. 506 pp.

Biging, G.S.; Dobbertin, M. 1995. Evaluation of competition indices in individual tree growth models. Forest Science, 41: 360-377.

Boivin, F.; Paquette, A.; Papaik, M.J.; Thiffault, N.; Messier, C. 2010. Do position and species identity of neighbours matter in 8-15-year-old post harvest mesic stands in the boreal mixedwood? Forest Ecology and Management, 260: 1124-1131.

Bragg, D.C. 2001. Potential relative increment (PRI): a new method to empirically derive optimal tree diameter growth. Ecological Modelling, 137: 77-92.

Choi, J.; Lorimer, C.G; Vanderwerker, J.; Cole, W.G.; Martin, G.L. 2001. A crown model for simulating long-term stand and gap dynamics in northern hard wood forests. Forest Ecology and Management, 152: 235-258.

Clark, D.A.; Clark, D.B. 2001. Getting the canopy: tree height growth in a neotropical rain forest. Ecology, 82: 1460-1472.

Contreras, M.A., Affleck, D.; Chung, W. 2011. Evaluating tree competition indices as predictors of basal area increment in western Montana forests. Forest Ecology and Management, 262: 1939-1949.

da Cunha, A.T. 2009. Increment modeling of individual Cedrela odorata trees in the Amazon forest. Dissertaçáo de Mestrado, Universidade Federal de Santa Maria, Santa Maria, Rio Grande do Sul. 87 pp (in Portuguese).

Daniels, R.F. 1976. Simple competition indices and their correlation with annual loblolly pine tree growth. Forest Science, 22: 454-456.

Daniels, R.F.; Burkhart, H.E.; Clason, T.R.A. 1986. comparison of competition measures for predicting growth of loblolly pine trees. Canadian Journal for Forest Research, 16: 1230-1237.

Das, A.; Battles, J.; Stephenson, N.L; Mantgem, P.J. 2011. The contribution of competition to tree mortality in old-growth coniferous forest. Forest Ecology and Management, 261: 1203 1213.

Davies, O.; Pommerening, A. 2008. The contribution of structural indices to the modelling of Sitka spruce (Picea sitchensis) and birch (Betula spp.) crowns. Forest Ecology and Management, 256: 68-77. 
Davis, L.S.; Johnson, K.N. 1987. Forest management, McGraw Hill, New York, USA. 790 pp.

Dawkins, H.C. 1963. Crown diameters: their relationship to bole diameter in tropical trees. Commonwealth Forest Review, 42: 318-333.

Dimov, L.D.; Chambers, J.L.; Lockhart, B.R. 2008. Five-year radial growth of red oaks in mixed bottomland hardwood stands. Forest Ecology and Management, 255: 2790-2800.

Dünisch, O.; Montoia, V.R.; Bauch, J. 2003. Dendroecological investigations on Swietenia macrophylla King and Cedrela odorata L. (Meliaceae) in the central Amazon. Trees-Structure and Function, 17: 244-250.

Durlo, M.A.; Denardi, L. 1998. Morphometry of Cabralea canjerana, in native secondary forest in Rio Grande do Sul, Ciência florestal, 8: 55-66 (in Portuguese).

Foli, E. G. 1993. Crown dimensions and diameter growth of some tropical mixed forest trees in Ghana. Tese de doutorado, University of Aberdeen, United of Kingdom, 185 pp. (in English).

Fox, J.C.; Bi, H.; Ades, P.K. 2008. Modelling spatial dependence in an irregular natural forest. Silva Fennica, 42: 35-48.

Freckleton, R.P.; Watkinson, A.R. 2001. Asymmetric competition between plant species, Functional Ecology, 15: 615-623.

Hasenauer, H. 2006. Sustainable forest management: Growth models for Europe. Springer, Berlin. 389 pp.

Holdaway, M.R. 1984. Modeling the effect of competition on tree diameter growth as applied in Stems. Forest Service - U.S. Department of Agriculture, Minnesota, USA. 12 pp.

Larocque, G.R. 2002. Examining different concepts for the development of a distance-dependent competition model for red pine diameter growth using long-term stand data differing in initial stand density. Forest Science, 48: 24-34.

Leary, R.A.; Holdaway, M.R. 1979. Modifier function. In: a generalized forest growth projection system applied to the Lake States Region. USDA Forest Service, General Technical Report.

Lieberman, M.; Lieberman, D. 1985. Simulation of growth curves from periodic increment data. Ecology, 66: 632-635.

Metcalf, C.J.E.; Clark, J.S.; Clark, D.A. 2009. Tree growth inference and prediction when the point of measurement changes: modeling around buttress in tropical forests. Journal of Tropical Ecology, 25: 1-12.

Mitsuda, Y.; Ito, S.; Takata, K. 2002. Effects of competitive and cooperative interaction among neighboring trees on tree growth in a naturally regenerated even-aged Larix sibirica Stand in considering height stratification. Journal of Forest Research, 7: 185-191.

Monserud, R.; Sterba, H. 1996. A basal area increment model for individual trees growing in even-and-uneven-aged forest stands in Austria. Forest Ecology and Management, 80: 57-80.

Muth, C.C.; Bazzaz, F.A. 2002. Tree canopy displacement at forest gap edges. Canadian Journal of Forestry Research, 32: 247-254.

Quicke, H.E.; Meldahl, R.S.; Kush, J.S. 1994. Basal area growth of individual trees: a model derived from a regional longleaf pine growth study. Forest Science, 40: 528-542.
Rinn, F. 2003. TSAP-Win, Version 4.64, reference manual: Time Series Analysis and Presentation Dendrochronology and Related Applications. Heidelberg. 110 pp.

SAS/STAT. 2004. User's guide - release 9.1.3 edition. Cary N.C.: SAS Institute Inc.

Schöngart, J. 2008. Growth-Oriented Logging (GOL): A new concept towards sustainable forest management in Central Amazonian várzea floodplains. Forest Ecology and Management, 256: 46-58.

Schröder, J.; Soalleiro, R.R.; Alonso, G.V. 2002. An age-independent basal area increment model for maritime pine trees in northwestern Spain. Forest Ecology and Management, 157: 55-64.

Schwinning, S.; Weiner, J. 1998. Mechanisms determining the degree of size asymmetry in competition among plants. Oecologia, 113 : 447-455.

Silva, J.N.M.; Carvalho, J.O.P; Lopes, J.C.A.; Almeida, B.F.; Costa, D.H.M.; Oliveira, L.C.; Vanclay, J.K.; Skovsgaard, J.P;. 1995. Growth and yield of a tropical rain in the Brazilian Amazon 13 years after logging, Forest Ecology and Management, 71: 267-274.

Sterba, H.; Blab, A.; Katzensteiner, K. 2002. Adapting an individual tree growth model for Norway Spruce (Picea abies L. Karst.) in pure and mixed species stands. Forest Ecology and Management, 159: 101-110.

Stokes, M.A.; Smiley, T.L. 1996. An Introduction to Tree-Ring Dating. The University of Arizona Press, Tucson. 73 pp.

Thorpe, H.C.; Astrup, R.; Trowbridge, A.; Coates, K.D. 2010. Competition and tree crowns: A neighbourhood analysis of three boreal tree species. Forest Ecology and Management, 259: 1586-1596.

Tonini, H. 2007. Índices de competição e o seu uso na modelagem do crescimento das árvores, Embrapa, BRA. 30 pp.

Vanclay, J.K. 1994. Modelling forest growth and yield: Applications to mixed tropical forests. CAB International, Copenhagen. $312 \mathrm{pp}$.

Vettenranta, J. 1999. Distance-dependent Models for Predicting the Development of Mixed Coniferous Forests in Finland. Silva Fennica, 33: 51-72.

West, P.W. 1995. Application of regression analysis to inventory data with measurements on successive occasions. Forest Ecology and Management, 71: 227-234.

Worbes, M. 1999. Annual growth rings, rainfall dependent growth and long-term growth patterns of tropical trees from the Forest Reserve Caparo in Venezuela. Journal of Ecology, 87: 391-403.

Wykoff, W.R. 1990. A basal area increment model for individual conifers in northern Rocky Mountains. Forest Science, 36: 1077-1104.

Zhang, S.; Amateis, R.L.; Burkhart, H.E. 1997. Constraining individual tree diameter increment and survival models for loblolly pine plantations. Forest Science, 43: 414-423.

Zhao, D.; Borders, B.; Wilson, M. 2004. Individual-tree diameter growth and mortality models for bottomland mixed-species hardwood stands in the lower Mississippi alluvial valley. Forest Ecology and Management, 199: 307-322.

Recebido em : 19-10-2011

Aceito em: 05-03-2012 